\title{
An automated food delivery system for behavioral and neurophysiological studies of learning and memory in freely moving monkeys
}

\author{
FRASER A. W. WILSON, BYOUNG-HOON KIM, JAE-WOOK RYOU, and YUAN-YE MA \\ Kunming Institute of Zoology, Chinese Academy of Sciences, Kunming, Yunan, China
}

\begin{abstract}
We describe a custom-built feeder based on stepping motor technology controlled by a laboratory computer. The feeder dispenses a wide range of foods: any fruit, vegetable, or nut. The feeder allows the investigator to reward monkeys with different foods within a single experimental day. The monkey's motivation to perform tasks is high and does not rely upon food regulation. The avoidance of regulation, as well as the palatability and variety of the rewards dispensed by our device, distinguishes it from commercially available products. We also describe the use of the feeder in the context of novel behavioral and neurophysiological studies in freely moving monkeys.
\end{abstract}

Behavioral studies in laboratory primates usually require the ability to reliably elicit the behavior under study by the delivery of a reinforcer. Most studies are carried out for fluid rewards because of the simplicity of fluid delivery. However, it can be advantageous to use food rewards in addition to fluids; in this way, investigators can use two different types of rewards and thus obtain good performance in two tasks (Wilson, Ma, Greenberg, Ryou, $\& \mathrm{Kim}, 2003)$. Independently of the type of reward, however, most studies require that food or water be controlled in order to motivate monkeys to perform a task, and consequently the health of the monkeys needs constant monitoring. The researcher, therefore, has to find a reinforcer that is both rewarding and healthy for the monkey, and this task is not easy to accomplish. For example, commercial feeders are limited in the types of foods they can deliver. Typically, the rewards are hard (e.g., peanuts and sugar-coated chocolate) and not well suited to a balanced diet. In addition, such reinforcers are relatively filling, and so limit the number of trials for which a monkey will work.

Accordingly, we developed a feeder (a motorized, computer-controlled turntable) that delivers small pieces of irregularly shaped nuts and soft juicy pieces of fruit and vegetables. These foods are both palatable and nutritious; there is little need for food regulation, since the monkeys are highly motivated to obtain these foods that supplement rather than substitute for their laboratory diet. Finally, we needed a device to reward monkeys per-

We appreciate the support of the NIH (Grant MH58415) and the Whitehall Foundation (Grant A89-04). Correspondence relating to this article may be sent to F. A. W. Wilson, Psychology 68, University of Arizona, 1503 East University Boulevard, Rm. 407, Tucson, AZ 85750 (e-mail: fraser@u.arizona.edu).

Note-This article was accepted by the previous editor, Jonathan Vaughan forming tasks as they moved freely in a specially designed testing room, and food delivery works well in this type of research.

\section{METHOD}

The feeders consist of several integrated components: a PC computer, the stepping motor components (motor, driver, and power supply; Intelligent Motion Systems [IMS], Marlborough, CT; www.imshome.com), and the feeder housing and carousels (which were built in-house). The PC controls the behavioral aspects of the experiments and runs TEMPO software (Reflective Computing, St. Louis, MO), a convenient interface for programming behavioral experiments and controlling external hardware/peripherals in the laboratory.

\section{Monkeys}

The 5 monkeys (Macaca mulatta) used in these studies were large (22.5-32.5 lb), were in excellent health, and gained weight as they grew during the study. They have worked with the present design of feeders for 4 years. In the holding colony, the monkeys were fed commercially available biscuits (Monkey Diet; Harlan Teklad, Indianapolis, IN). Monkey food preferences were established by presenting them with many different sorts of fruits, vegetables, and nuts. These foods (e.g., 1/8 grape, apple piece, $1 / 2$ peanut, raisin) were freshly prepared every day and loaded into the feeder before each experiment. The monkeys were trained to retrieve food by demonstrations from investigators. Once they saw that lifting the carousel lids revealed pieces of food, the monkeys began to initiate lid opening spontaneously. Efficient retrieval required the monkeys to sit before opening the feeder lid. The monkeys usually used one hand to raise the lid and the other to retrieve the food. This skill required good visuomotor coordination and took several 
days to learn, in part because the monkeys showed some caution as a result of the movements of the carousel inside the feeder housing. All procedures were carried out in accordance with the National Institutes of Health's Guide for the Care and Use of Laboratory Animals (NIH Publication No. 80-23) and with the guidelines of the University of Arizona's IACUC committee.

\section{Behavioral Tasks}

The monkeys performed a variety of memory guided (Ma, Ryou, Kim, \& Wilson, 2003; Ryou et al., 2004; Ryou \& Wilson, 2004) and visually guided tasks in which they moved between the four feeders in a testing room $(16 \times 16 \mathrm{ft})$ specifically built for behavioral experiments. The feeders were located in the center of each wall; the distance between the fronts of adjacent boxes was $\sim 7 \mathrm{ft}$. A typical task was a variant of the spatial delayed alternation task (Stamm, 1987), in which the monkeys walked between three of the four boxes in sequence (e.g., west $\rightarrow$ north $\rightarrow$ east $\rightarrow$ north $\rightarrow$ west, etc.). This task was guided by memory for the previous behavioral choice. The task was difficult, but performance was usually better than $95 \%$ correct for 150 trials/day. Our Web site includes a movie that demonstrates performance of the task and use of the feeder (www.arizona.edu/ primate/).

\section{Hardware}

Computer. A generic PC (Pentium 133, 16 MB RAM) was used to control the behavioral aspects of the experiments - that is, trial selection, sensing of feeder lid position, generation of visual and auditory cues to guide the monkeys, and commands to the stepping motor driver. A dedicated I/O card (Model DIO-96; Measurement Computing [formerly ComputerBoards, Inc.], Middleboro, MA) was housed in the PC; its 96 lines could be configured flexibly to either read or write TTL-level signals.

Feeders. These consisted of a housing mounted on legs so that the top surface was 18 in. from ground level. The entire top surface of the feeder housing was hinged so that it could be held open during loading by the investigator (Figure 1). When this top surface was closed, food was retrieved by lifting a small hinged lid at the front of the feeder. This lid was weighted with a magnet (see below) that also served as a handle for the monkey. Opening the lid revealed a slot in the top surface and the underlying carousel compartment in which the food was placed. When the lid was raised, only one food item within a single carousel compartment could be seen. A stopper placed behind the lid prevented it from being raised beyond $80^{\circ}$, and thus when the lid was released it fell back to horizontal.

Each feeder delivered 50 rewards per session and had several notable features: (1) a bright white LED (to identify the current target); (2) a loudspeaker (an auditory cue signaled a behavioral error); and (3) a feeder lid with a magnet, with a corresponding magnetically driven switch (Radio Shack 49-496) in the feeder wall close to the lid. The lid had to be lifted in order to actuate the stepping
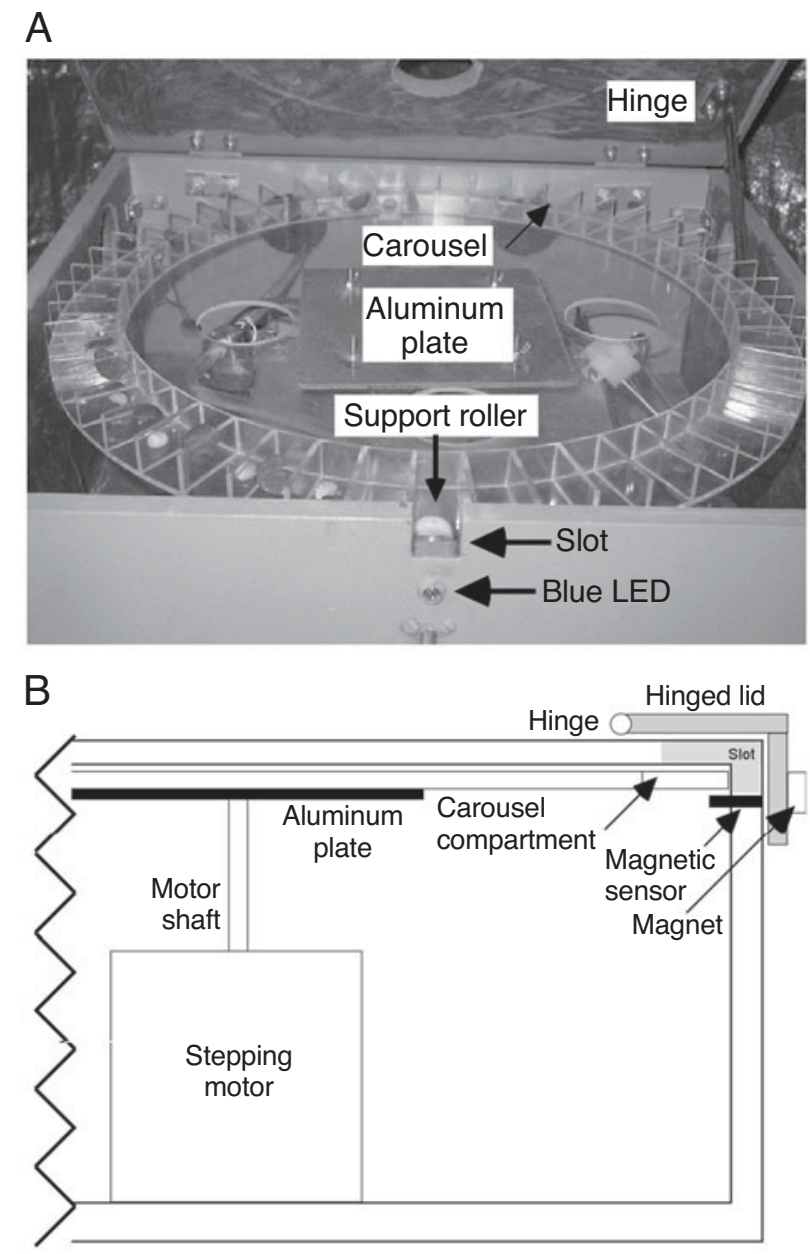

Figure 1. (A) Front view of feeder with top surface raised to load foods. In this experiment, pieces of grape, raisin, and nut were used as rewards. (B) Partial side view of feeder showing the slot, the lid, and the positions of the magnet and the magnetically driven switch.

motor and food delivery. When the lid (and the magnet) were lifted, the switch responded to the loss of magnetic field strength by opening, allowing the detection of a TTL-level signal by the I/O card in the computer. This signal was interpreted by the control software (e.g., is the correct or the wrong lid open?), which decided whether or not to send the stepping command to the driver.

Each carousel had 50 compartments, with the compartment mouths subtending $\sim 7.2^{\circ}$. A single piece of food was placed at the back of each compartment. The carousels were made of three polycarbonate components: a disk, rectangular walls forming the sides of the food compartments, and a strip that formed the rear wall of each compartment. Each carousel, including disk, compartments, and rear wall, weighed $\sim 1.3 \mathrm{lb}$. The carousels were supported in part by two rollers placed under the slot and rear wall; the rollers prevented the carousels from being pushed downward from the horizontal when food was being retrieved. 
Stepping motors. The stepping motors were bolted to the floor of the housing. A shaft from the stepping motor was coupled to a horizontal aluminum plate to which the carousel was bolted. As the carousel rotated, the mouth of each compartment moved into alignment with the slot in the front of the housing, so that food was easily removed once the lid was open (Figure 1A).

The resolution of the stepping motor was $0.9^{\circ}$ of rotation per step in half-step mode. On the basis of the width of the monkeys' fingers, a reasonable number of trials, and limits on the size of the feeder housing, we determined that a suitable width of the food compartments was $\sim 7 / 8$ in. In order to obtain 50 operations (reward deliveries) per feeder within a testing session, the carousel disks had a diameter of 16.5 in.

Since our system does not have a feedback system for finding a start position for the carousels, we aligned one compartment with the housing's slot (Figure 1) before the testing session, when the carousel was free to rotate. Once aligned, the carousel delivered all rewards in a session without error. When the testing session began, the stepping motors were on continuously in order to lock the stepping motors until the signal was given to move the carousels.

The stepping motor components obtained from IMS included one 40-V power supply (Model IP406), four stepping motor drivers (Model IB462), and four stepping motors (Model M2-3424-T). We have used the stepping motors daily for 4 years. With the exception of a failure of one driver (which required a replacement), the systems have worked both reliably and flawlessly.

\section{Control of the Stepping Motors}

For our purposes, operation of the stepping motors required three control signals for selection of half-stepping mode, counterclockwise direction, and amplitude (number of pulses). The IB462 driver has an interface that responds to TTL-level signals appropriate for each of these pieces of information; specifically, a TTL low keeps the driver in half-step mode, and a pulse train of 8 TTL highs (20-msec pulses with 20-msec interpulse intervals) moves the stepper. We selected half-step mode in order to make the $7.2^{\circ}$ rotations.

The stepping drivers generate a lot of heat $\left(80^{\circ} \mathrm{C}\right.$ is the maximum recommended). This level of heat is normal for the driver, but we used a fan to dissipate the heat. IMS also provides controls to limit current and heat sinks that are probably crucial to allow the motors to function for many hours daily and to protect them from damage by power shocks.

\section{Neurophysiology}

The IP406 power supply works well for studies in which the control of behavior was the only consideration. However, it has the disadvantage of producing large electrical artifacts that interfere with neurophysiological recordings. The artifacts become observable when monkeys (and their head-mounted recording amplifiers; Wilson et al., 2003) are close to the stepping motor.
In order to eliminate these artifacts from the stepping system during neurophysiological experiments, we did the following: (1) We used a $12-\mathrm{V}$ DC car battery to power the stepping motors and drivers. This battery was recharged conventionally. (2) The motors were encapsulated in galvanized iron shielding, and the feeder housing was coated inside with nickel paint in order to provide an electrical shield. These two shields were connected to the power supply ground. (3) The helmets worn by the monkeys were coated inside with nickel paint and were electrically connected to the power supply ground.

Figure 2 shows examples of neuronal activity related to the opening of the feeder lids. The neuron was strongly responsive when the left hand was used but was much less responsive when the right hand was used. The data from Figure 2A show trials when the east feeder was being opened with the left or the right hand. Figure $2 \mathrm{~B}$ graphs the responses for all eight trial types (four with the left hand, $M=29.6$ spikes/sec; four with the right hand, $M=6$ spikes/sec). Neurons were thus selectively active when the left hand was used. If artifacts had been present during actuation of the feeders, one would have

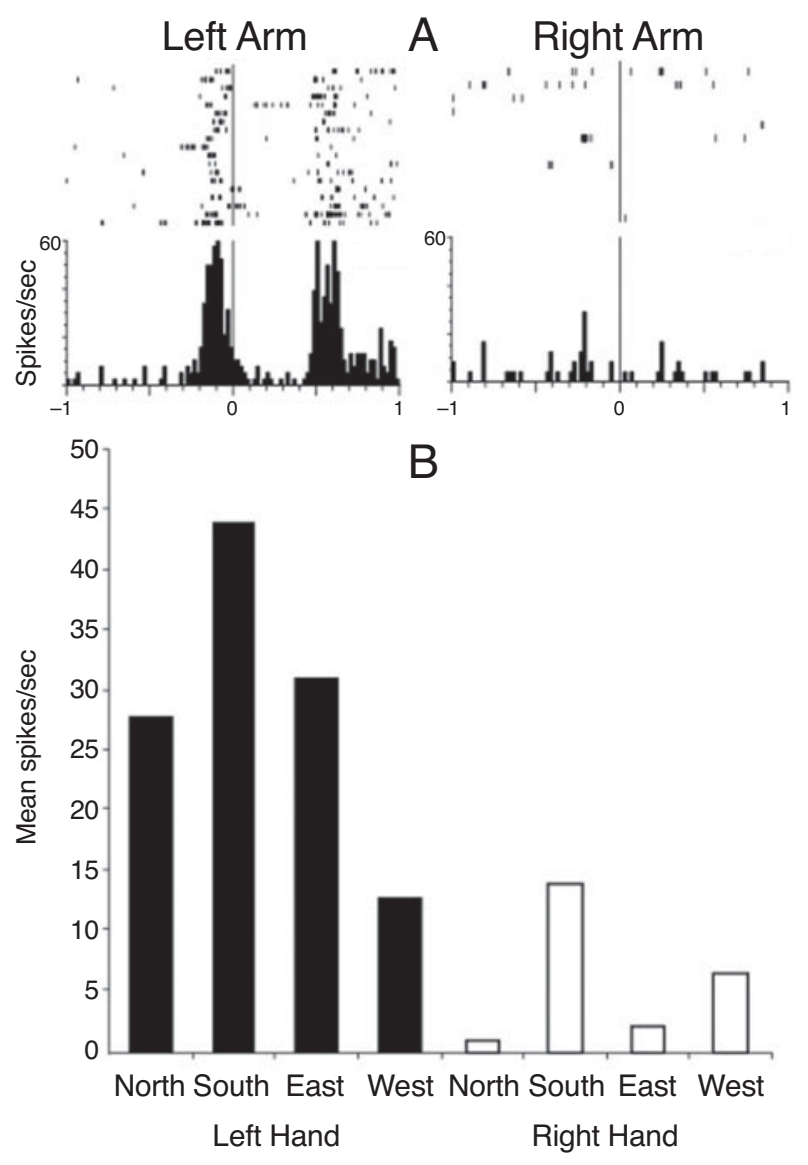

Figure 2. Neuronal responses related to opening the feeder lids. (A) Trials in which the east feeder lid was opened. Those in which the left hand was used are separated from ones in which the right hand was used. Time zero $=$ moment of lid opening. (B) All eight trial types, in which each hand was used at each of the four feeders. 
expected the neuronal responses to occur at time zero for $\sim 500 \mathrm{msec}$.

\section{Construction}

For an illustration of the fully built feeder carousel unit, please refer once again to Figure 1.

Carousel. The polycarbonate disk (diameter, $16^{1 / 2} \mathrm{in}$.; thickness, $1 / 8$ in.) and the rectangular walls of the compartments $\left(1 \frac{1}{2}\right.$ in. long $\times 3 / 4$ in. high) were cut to shape and size by a local plastics company. To create the partitions, we used software (Canvas 8 for Windows; ACD Systems of America, www.acdamerica.com) to draw a template in the form of a star pattern in which the angular distance subtended between two adjacent radii was $7.2^{\circ}$ ( $7 / 8$ in. wide $\times 1 \frac{1}{2}$ in. deep). The pattern was printed and taped to the carousel base in order to mark the position of each compartment wall on the base. The rectangular side walls were then bonded to the disk with WELD*ON 5 (IPS Corp., Compton, CA). In 4 years of daily operation, only 4 of 200 walls have separated from the disk. A continuous strip of polycarbonate was cemented to the disk to form the rear walls of the compartments.

Feeder housing. Mounted on 10-in. legs, the housing ( 8 in. high $\times 18$ in. wide $\times 18$ in. deep) is a box made of particle board, well sealed with paint to comply with NIH guidelines. All components are bolted internally with angle brackets. The front of the top surface of the housing has a weighted, hinged lid $(2 \times 2$ in. $)$. Opening the lid reveals a slot ( $7 / 8$ in. wide $\times 1 \frac{1}{2}$ in. deep) and the underlying carousel compartment in which food is placed. Internal surfaces of the housing are coated with conductive paint to provide electrical insulation for neurophysiological studies. The 10-in. legs (polypropylene angle; McMaster-Carr, Elmhurst, IL) are attached with wood screws.

Coupling the carousel to the stepper motor. Each motor has a protruding 11/4-in. cylindrical shaft; the shaft rotates and moves the carousel incrementally. The carousel is coupled to the motor shaft with a horizontal aluminum plate $(6 \times 6 \times 3 / 16$ in.) from which protrudes a 1/1/4-in. cylindrical shaft. The two shafts are coupled with thickwalled Tygon tubing and secured with two hose clamps. The aluminum plate is drilled and tapped so that four bolts can be screwed into it. The bolts project upward so that the carousel base (which is drilled to accept the bolts) sits on the aluminum plate and is locked with wing nuts. This assembly is easily disassembled for cleaning. The coupling has remained secure since the original construction. The carousel floats above the motor and is supported vertically by two rollers.

\section{DISCUSSION}

The feeder is reliable and simple to build, given the expertise of most laboratories with computers and the ease of controlling peripheral devices such as stepping motors. The chief advantage of our feeder is the wide range of foods it delivers, which can be changed to suit the preferences of individual animals. Since monkeys are highly motivated to perform tasks by favorite foods, it is unnecessary to regulate their dietary intake for simple tasks, although this will depend in part on the difficulty of a task. This issue is important, because most investigators do find it necessary to impose dietary restrictions on their animals. NIH guidelines stipulate that animals in behavioral studies must not lose weight beyond $15 \%$ of the average, but this problem is obviated by our device; our monkeys are very well fed, since they eat a standard laboratory diet that is supplemented daily with fruits, vegetables, and nuts during laboratory testing. After 4 years of work with the device, the monkeys have gained weight normally, are in good health, and are even inclined to become slightly obese under the feeding regimen, despite the balanced diet and exercise in a testing room that allows the monkeys to move freely.

From an investigator's point of view, the feeder has two limitations: The number of food items per carousel is relatively small, and the food has to be prepared and loaded. Certain monkeys will work for 300 trials (but typically less if the task is demanding); in such long sessions, monkeys must be returned to their transport chair while food reloading takes place. A recently developed and commercially available feeder (see Mitz, Boring, Wise, \& Lebedev, 2001) is capable of delivering 500 rewards. However, commercial devices usually require dieting for monkeys. Accordingly, we think the health benefits of the feeder we describe are worth its consideration.

We have also found that monkeys working for food rewards will also work for fluid rewards in a hand movement task while seated in a primate chair. Thus, one benefit of using foods is that an investigator can also study other behaviors using juice rewards on the same day.

The large majority of neurophysiological studies are carried out while monkeys are seated in chairs. It is rare to find studies in which monkeys are able to move about their environment (but see Ludvig, Botero, Tang, Gohil, \& Kral, 2001; Rolls, Robertson, \& Georges-François, 1997). The emphasis on using chaired monkeys clearly follows from the difficulties of melding behavioral and neurophysiological techniques in unrestrained animals. However, the ability to study unimpeded movement affords tremendous advantages in understanding the relationship between brain and behavior, and thus we have developed the necessary methods for studying behavior in freely moving monkeys while simultaneously measuring neuronal activity. Our feeders are a marked improvement over other systems simply because they avoid the health problems associated with food or water regulation.

\section{REFERENCES}

Ludvig, N., Botero, J. M., Tang, H. M., Gohil, B., \& Kral, J. G. (2001). Single-cell recording from the brain of freely moving monkeys. Journal of Neuroscience Methods, 106, 179-187.

MA, Y.-Y., RYou, J.-W., KIM, B.-H., \& WiLson, F. A. W. (2003). Spatiallydirected movement and neuronal activity in freely moving monkeys. In S. Mori, D. G. Stuart, \& M. Wiesendanger (Eds.), Brain mechanisms for the integration of posture and movement (pp. 505-512). Amsterdam: Elsevier.

Mitz, A. R., Boring, S. A., Wise, S. P., \& Lebedev, M. A. (2001). A novel 
food-delivery device for neurophysiological and neuropsychological studies in monkeys. Journal of Neuroscience Methods, 109, 129-135. Rolls, E. T., Robertson, R. G., \& Georges-François, P. (1997). Spatial view cells in the primate hippocampus. European Journal of Neuroscience, 9, 1789-1794.

Ryou, J.-W., Kim, B.-H., SeoK, B., Lim, S. L., Lamendola, N., Ma, Y.-Y., \& WiLson, F. A. W. (2004). Dissociation of spatial functions in dorsolateral prefrontal cortex and hippocampus in freely moving monkeys. Manuscript submitted for publication.

Ryou, J.-W., \& WILSON, F. A. W. (2004). Making your next move: Dor- solateral prefrontal cortex and planning a sequence of actions in freely moving monkeys. Cognitive, Affective, \& Behavioral Neuroscience, 4, 430-443.

Stamm, J. S. (1987). The riddle of the monkey's delayed-response deficit has been solved. In E. Perecman (Ed.), The frontal lobes revisited (pp. 73-89). New York: IRBN.

Wilson, F. A. W., Ma, Y.-Y., Greenberg, P. A., Ryou, J.-W., \& Kim, B.-H. (2003). A microelectrode drive for long term recording of neurons in freely moving and chaired monkeys. Journal of Neuroscience Methods, 127, 49-61.

\section{APPENDIX}

The following costs were incurred in building four feeders. Intelligent Motion Systems (www.imshome.com) sells the stepper motors and associated items. A single $40-\mathrm{V}$ power supply is sufficient for four motors.

$\begin{array}{lr}\text { IB462 stepping motor driver } & \$ 83.50 * 4=\$ 334 \\ \text { M2-3424-T hybrid stepping motor } & \$ 145 * 4=\$ 580 \\ \text { IP406 40-V power supply } & \$ 139 \\ \text { Polycarbonate materials and machining } & \$ 110 \\ \text { Measurement Computing DIO-96 I/O card } & \$ 180\end{array}$

Software for control of the behavioral task and the stepping motors is available from the authors or can be downloaded from the laboratory Web site (www.arizona.edu/ primate/).

(Manuscript received December 4, 2003;

revision accepted for publication August 9, 2004.) 